\title{
Radiative effects in deep virtual Compton scattering
}

\author{
Igor Akushevich $^{*}$ \\ Physics Department, Duke University, Durham, North Carolina 27708, USA \\ Alexander Ilyichev ${ }^{\dagger}$ \\ Institute for Nuclear Problems, Belarusian State University, Minsk 220030, Belarus
}

(Received 4 December 2017; published 17 July 2018)

\begin{abstract}
Radiative corrections to the cross sections of photon electroproduction and the single spin asymmetries induced by the interference between the Bethe-Heitler and deep virtual Compton scattering amplitudes are calculated within the leading log approximation. The deep virtual Compton scattering amplitude is presented in the Belitsky-Müller-Kirchner (BMK) approximation for the polarized initial particles. The Fortran code for estimation of the radiative effects in a given kinematic point and Monte Carlo generator for simulation of one or two photons are developed. Numerical results are performed for beam-spin asymmetries in kinematical conditions of current experiments in the Jefferson Laboratory.
\end{abstract}

DOI: 10.1103/PhysRevD.98.013005

\section{INTRODUCTION}

The process of deep virtual Compton scattering (DVCS) is considered to provide useful information for extraction of properties of the generalized parton distributions. Experimentally DVCS is investigated through the measurements of the cross section and asymmetries in the processes of the photon electroproduction with both an unpolarized and polarized electron beam and proton target. Three Feynman graphs presented in Fig. 1 contribute to the cross section of the photon electroproduction. Graphs (a) and (b) represent the amplitude of the Bethe-Heitler (BH) process and graph (c) describes the DVCS amplitude. The latter gives the access to the properties of the generalized parton distributions; therefore, it is of specific interest. During the last decade, the process was intensively investigated both theoretically [1,2] and experimentally [3-6]. The cross section of the photon electroproduction is dominated by the $\mathrm{BH}$ process, i.e., by the sum of two BH amplitudes [graphs (a) and (b)] squared. Therefore, to get access to the DVCS process the researcher has to find an asymmetry vanishing for a pure $\mathrm{BH}$ process and for which the main contribution would involve the DVCS amplitude.

\footnotetext{
*Also at Jefferson Lab., Newport News, VA 23606, USA. igor.akushevich@duke.edu

ily@hep.by

Published by the American Physical Society under the terms of the Creative Commons Attribution 4.0 International license. Further distribution of this work must maintain attribution to the author(s) and the published article's title, journal citation, and DOI. Funded by SCOAP ${ }^{3}$.
}

The well-known example of an appropriate asymmetry is the single beam-spin asymmetry.

The QED radiative correction (RC) is one serious source of systematical uncertainties and therefore must be known with any predetermined accuracy. Available calculations of QED radiative effects in [7-10] are focused on $\mathrm{RC}$ to $\mathrm{BH}$ process or have certain limitations and cannot cover needs of modern requirements on the photon electroproduction. In this paper we present the radiative correction calculations to the cross section of BH and DVCS processes in leading approximation. In the approximation the only leading term containing $L=\log \left(Q^{2} / \mathrm{m}^{2}\right)$ ( $m$ is the electron mass) is kept. Since the structure of the dependence of the RC cross section on the electron mass is

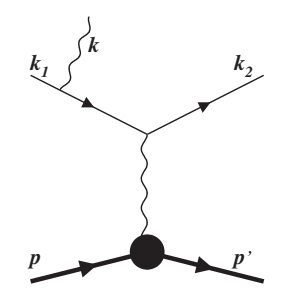

(a)

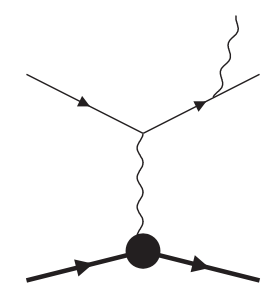

(b)

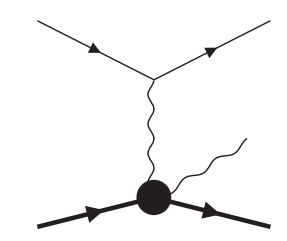

(c)

FIG. 1. Feynman graphs of BH (a) and (b) and DVCS process (c). 
$\sigma_{\mathrm{RC}}=A \log \left(Q^{2} / m^{2}\right)+B+O\left(m^{2} / Q^{2}\right)$, where $A$ and $B$ do not depend on the electron mass, the used approximation allows us to keep the major part of the RC, e.g., for experiments with transferring momentum squared of one $\mathrm{GeV}$ squared $\left(Q^{2} \sim 1 \mathrm{GeV}^{2}\right), \log \left(Q^{2} / m^{2}\right) \sim 15$.

Calculation of RC for $\mathrm{BH}$ and DVCS requires knowledge of the hadronic structure for these processes. Although the $\mathrm{BH}$ cross section can be described in the model-independent way using the nucleon form factors, the description of DVCS amplitude requires assumptions on a model for hadronic subprocesses. A reasonable approach leading to analytic and transparent results is the BMK approximation [11] in which quantitative estimates for the azimuthal and spin asymmetries can be obtained relying on a simple ansatz for the generalized parton distributions. The theoretical consideration went beyond the leading twist and involved the complete analysis in the twist-three approximation. In practice the results obtained in the BMK approximation are considered valid for small squared of the transferred momentum between initial and final proton $t,-t \leq 0.5 \mathrm{GeV}^{2}$.

The paper is organized as follows. The lowest order contribution to the cross section for the photon electroproduction induced by the interference of $\mathrm{BH}$ and DVCS amplitudes is represented in Sec. II. Specific attention is paid on explicit representation of the cross section including the polarization part of the cross section, mass corrections, as well as the angular structure of the cross section. RC calculation is performed in Sec. III. First, we calculate the matrix element squared and trace all sources of occurrence of the electron mass dependence. We keep the contributions of $\mathrm{BH}$ amplitude squared and the interference between the BH and DVCS amplitudes, and drop the pure DVCS contribution. Second, we represent the phase space of two final photons, introduce so-called shifted kinematics, and calculate integrals over additional photon phase space. Third, we add the contribution of loops and calculate the lowest order RC to the cross section. Fourth, we generalize the result for the $\mathrm{RC}$ to the cross section from the $\mathrm{BH}$ and DVCS interference to represent the higher order corrections. Section IV presents the codes for numerical calculation of $\mathrm{RC}$ in a kinematical point and the Monte Carlo generator allowing for simulating the events with one or two radiated photon(s). Section V provides numeric estimates of the radiative effects in current experiments in JLab focusing on the $\mathrm{RC}$ to the cross section in a wide kinematic region of modern experiments at JLab. Finally, in Sec. VI we summarize the results obtained in this paper.

\section{THE CROSS SECTION OF THE INTERFERENCE OF BH AND DVCS AMPLITUDES}

The process of interest is

$$
e\left(k_{1}, \xi\right)+p(p, \eta) \rightarrow e^{\prime}\left(k_{2}\right)+p^{\prime}\left(p^{\prime}\right)+\gamma(k),
$$

where $k_{1}^{2}=k_{2}^{2}=m^{2}, p^{2}=p^{2}=M^{2}, k^{2}=0, \xi$ and $\eta$ are polarization vectors of the initial lepton and proton, and $m$ and $M$ are their masses, respectively. The process (1) is traditionally described by the five kinematical variables: $S=2 k_{1} p, \quad Q^{2}=-\left(k_{1}-k_{2}\right)^{2}, \quad x=Q^{2} /\left(2 p\left(k_{1}-k_{2}\right)\right)$, $t=\left(p-p^{\prime}\right)^{2}$, and $\phi$ [the angle between $\left(\mathbf{k}_{\mathbf{1}}, \mathbf{k}_{\mathbf{2}}\right)$ and $\left(\mathbf{q}, \mathbf{p}^{\prime}\right)$ planes, $q=k_{1}-k_{2}$ ]. Four latter variables involve the momenta of final particles; therefore, the cross section of interest is $\sigma \equiv d \sigma_{0} / d Q^{2} d x d t d \phi$. Because of azimuthal symmetry, the integration over lepton angle $\phi_{e}$ [i.e., the angle between $\left(\mathbf{k}_{\mathbf{1}}, \mathbf{k}_{\mathbf{2}}\right)$ and $\left(\mathbf{q}, \mathbf{k}_{\mathbf{2}}\right)$ planes] has been completed in this cross section. The symmetry can be violated in the case of transversal target polarization; therefore, the five dimensional cross section is considered $\sigma \equiv d \sigma_{0} / d Q^{2} d x d t d \phi\left(d \phi_{e} / 2 \pi\right)$ in this case. Explicitly, the cross section of the photon electroproduction involving the contributions of $\mathrm{BH}$ and DVCS interference is

$$
d \sigma_{1 \gamma}=\frac{1}{2 S} \mathcal{M}_{1 \gamma}^{2} d \Gamma_{0}
$$

Phase space for the photon electroproduction cross section is parametrized as

$$
\begin{aligned}
d \Gamma_{0} & =\frac{1}{(2 \pi)^{5}} \frac{d^{3} k_{2}}{2 E_{2}} \frac{d^{3} p^{\prime}}{2 p_{0}^{\prime}} \frac{d^{3} k}{2 \omega} \delta^{4}\left(k_{1}+p-k_{2}-p^{\prime}-k\right) \\
& =\frac{Q^{2} d Q^{2} d x d t d \phi}{(4 \pi)^{4} x^{2} S \sqrt{\lambda_{Y}}}
\end{aligned}
$$

with $\lambda_{Y}=S_{x}^{2}+4 M^{2} Q^{2}$ and $S_{x}=S-X=Q^{2} / x$. Kinematical limits on $t$ are defined as

$$
t_{2,1}=-\frac{1}{2 W^{2}}\left(\left(S_{x}-Q^{2}\right)\left(S_{x} \pm \sqrt{\lambda_{Y}}\right)+2 M^{2} Q^{2}\right),
$$

where $W^{2}=S_{x}-Q^{2}+M^{2}$.

All variables are ultimately expressed in terms of the five kinematical variables: $S, t, Q^{2}, x$ and $\phi$, e.g.,

$$
\begin{aligned}
w_{0}= & 2 k k_{1}=-\frac{1}{2}\left(t+Q^{2}\right)+\frac{S_{p}}{2 \lambda_{Y}}\left(S_{x}\left(Q^{2}-t\right)+2 t Q^{2}\right) \\
& +\frac{\sqrt{\lambda_{u w}}}{\lambda_{Y}} \cos \phi, \\
u_{0}= & 2 k k_{2}=w_{0}+Q^{2}+t
\end{aligned}
$$

with $S_{p}=S+X$ and

$\lambda_{u w}=4 W^{2}\left(Q^{2}\left(S X-M^{2} Q^{2}\right)-m^{2} \lambda_{Y}\right)\left(t-t_{1}\right)\left(t_{2}-t\right)$.

Note that in massless approximation (for $m \rightarrow 0$ ) the cross section of $\mathrm{BH}$ and DVCS interference exactly coincides with the results of [11]. The following equations relating our notation to the notation of Ref. [11] [Eqs. (30) and (32)] 
are valid: $u_{0}=\mathcal{P}_{2} Q^{2}, \quad w_{0}=-\mathcal{P}_{1} Q^{2}$, and (for $m \rightarrow 0$ ) $\lambda_{u w}=4 Q^{4} S^{2} S_{x}^{2} K^{2}$

The interference term of matrix element squared is

$$
\mathcal{M}_{1 \gamma}^{2}=\left(\mathcal{M}_{1}^{l}+\mathcal{M}_{2}^{l}\right) \mathcal{M}^{h^{\dagger}}+\mathcal{M}^{h}\left(\mathcal{M}_{1}^{l}+\mathcal{M}_{2}^{l}\right)^{\dagger},
$$

where $\mathcal{M}_{1}^{l}$ and $\mathcal{M}_{2}^{l}$ corresponds to diagrams presented in Figs. 1(a) and 1(b), and $\mathcal{M}^{h}$ described hadronic emission contribution presented in Fig. 1(c). The BH matrix element is $\mathcal{M}_{\mathrm{BH}}=\mathcal{M}_{1}^{l}+\mathcal{M}_{2}^{l}=e^{3} t^{-1} J_{\mu}^{h} J_{\mu \alpha}^{\mathrm{BH}}\left(k_{1}, k_{2}, k\right) \epsilon^{\alpha}$ with

$$
\begin{aligned}
J_{\mu \alpha}^{\mathrm{BH}}\left(k_{1}, k_{2}, k\right) & =J_{\mu \alpha}^{\mathrm{BH} 1}\left(k_{1}, k\right)+J_{\mu \alpha}^{\mathrm{BH} 2}\left(k_{2}, k\right), \\
J_{\mu}^{h} & =\bar{u}\left(p^{\prime}\right)\left(\gamma_{\mu} F_{1}+i \sigma_{\mu \nu} \frac{p_{\nu}^{\prime}-p_{\nu}}{2 M} F_{2}\right) u(p)
\end{aligned}
$$

and

$$
\begin{aligned}
J_{\mu \alpha}^{\mathrm{BH}}\left(k_{1}, k_{2}, k\right) & =\bar{u}_{2}\left[\gamma_{\mu} \frac{\hat{k}_{1}-\hat{k}+m}{-2 k k_{1}} \gamma_{\alpha}+\gamma_{\alpha} \frac{\hat{k}_{2}+\hat{k}+m}{2 k k_{2}} \gamma_{\mu}\right] u_{1} \\
& =-\bar{u}_{2}\left[\left(\frac{k_{1 \alpha}}{k k_{1}}-\frac{k_{2 \alpha}}{k k_{2}}\right) \gamma_{\mu}-\frac{\gamma_{\mu} \hat{k} \gamma_{\alpha}}{2 k k_{1}}-\frac{\gamma_{\alpha} \hat{k} \gamma_{\mu}}{2 k k_{2}}\right] u_{1} .
\end{aligned}
$$

Here $\bar{u}_{2} \equiv \bar{u}\left(k_{2}\right), u_{1} \equiv u\left(k_{1}\right)$, and $\epsilon$ is the photon polarization vector. The matrix element $\mathcal{M}_{\mathrm{BH}}$ corresponds to the graphs in Figs. 1(a) and 1(b).

The DVCS amplitude is calculated in [11]. The set of explicit formulas [e.g., Eqs. (1)-(8)] allows us to present the DVCS amplitude in terms of covariant hadronic structures and the Compton form factors. This representation is appropriate for $\mathrm{RC}$ calculation. As a result the interference (7) has a form:

$$
\begin{aligned}
& \mathcal{M}_{1 \gamma}^{2}\left(k_{1}, k_{2}, k\right) \\
& =\frac{64 \pi^{3} \alpha^{3}}{t Q^{2}}\left(J_{\mu}^{h} J_{\mu \alpha}^{\mathrm{BH}}\left(k_{1}, k_{2}, k\right)\left(T_{\alpha \nu}(k) J_{\nu}^{(0)}\left(k_{1}, k_{2}\right)\right)^{\dagger}\right. \\
& \left.\quad+T_{\alpha \nu}(k) J_{\nu}^{(0)}\left(k_{1}, k_{2}\right)\left(J_{\mu}^{h} J_{\mu \alpha}^{\mathrm{BH}}\left(k_{1}, k_{2}, k\right)\right)^{\dagger}\right) .
\end{aligned}
$$

Here $J_{\mu}^{(0)}\left(k_{1}, k_{2}\right)=\bar{u}_{2} \gamma_{\mu} u_{1}$ and $T_{\nu \mu}(k)$ are defined by Eqs. (1)-(8) of [11].

We calculate the cross section in the BMK approximation. In this approximation the cross section is represented through the sum over the finite number of terms reflecting the $\phi$-dependence. Respective coefficients are referred as the Fourier coefficients. They are calculated in the leading approximation at $M^{2}$ and $t$ simultaneously going to zero. We present them in the form appropriate for further RC calculation.

The most important observable quantity is the beam-spin asymmetry:

$$
A_{1 \gamma}=\frac{\sigma_{I}^{p}}{\sigma_{\mathrm{BH}}^{u}+\sigma_{I}^{u}},
$$

where $\sigma_{\mathrm{BH}}^{u}$ is the $\mathrm{BH}$ cross section of unpolarized electrons and protons, and $\sigma_{I}^{u, p}$ are unpolarized and spin-dependent parts of the cross section that resulted from the interference of the BH and DVCS amplitudes.

In BMK approximation, the $\mathrm{BH}$ cross section is expressed as

$$
\sigma_{\mathrm{BH}}^{u}=\frac{f_{\mathrm{BH}}}{\mathcal{P}_{1} \mathcal{P}_{2}}\left(c_{0}^{\mathrm{BH}}+c_{1}^{\mathrm{BH}} \cos \phi+c_{2}^{\mathrm{BH}} \cos 2 \phi\right),
$$

where $f_{\mathrm{BH}}=\alpha^{3} S_{x}^{3} /\left(8 \pi x^{3} t \lambda_{Y}^{5 / 2}\right)$. The four and two nonzero coefficients for unpolarized and polarized beam, respectively:

$$
\begin{aligned}
\sigma_{I}^{u} & =\frac{f_{I}}{\mathcal{P}_{1} \mathcal{P}_{2}}\left(c_{0}^{I}+c_{1}^{I} \cos \phi+c_{2}^{I} \cos 2 \phi+c_{3}^{I} \cos 3 \phi\right), \\
\sigma_{I}^{p} & =\frac{f_{I}}{\mathcal{P}_{1} \mathcal{P}_{2}}\left(s_{1}^{I} \sin \phi+s_{2}^{I} \sin 2 \phi\right)
\end{aligned}
$$

with $f_{I}=\alpha^{3} S /\left(8 \pi Q^{4} t \lambda_{Y}^{1 / 2}\right)$. The corresponding Fourier coefficients $c_{i}$ and $s_{i}$ are calculated in [11].

\section{RC CROSS SECTION}

The cross section of two-photon emission, i.e., the process,

$e\left(k_{1}\right)+p(p) \rightarrow e^{\prime}\left(k_{2}\right)+p^{\prime}\left(p^{\prime}\right)+\gamma\left(\kappa_{1}\right)+\gamma\left(\kappa_{2}\right)$,

is proportional to the matrix element squared of the process with two real photons in the final state that has the contribution of pure leptonic correction [shown in Figs. 2(a)-2(c) and discussed in [9] ] and the contribution of the interference between lepton and hadron emissions. The interference $\left(\mathcal{M}_{2 \gamma}^{2}\right)$ contributes $\mathrm{RC}$ to the cross section (2). The cross section of RC due to two-photon emission is

$$
d \sigma=\frac{1}{4 S} \mathcal{M}_{2 \gamma}^{2} d \Gamma
$$

where

$$
\begin{aligned}
d \Gamma= & \frac{1}{(2 \pi)^{8}} \frac{d^{3} k_{2}}{2 E_{2}} \frac{d^{3} p^{\prime}}{2 p_{0}^{\prime}} \frac{d^{3} \kappa_{1}}{2 \omega_{1}} \frac{d^{3} \kappa_{2}}{2 \omega_{2}} \\
& \times \delta^{4}\left(k_{1}+p-k_{2}-p^{\prime}-\kappa_{1}-\kappa_{2}\right)
\end{aligned}
$$

and the additional factor of 2 in the denominator is because there are two identical particles (photons) in the final state. The matrix element squared $\mathcal{M}_{2 \gamma}^{2}$ is 


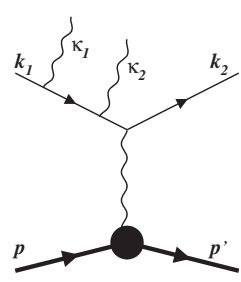

(a)

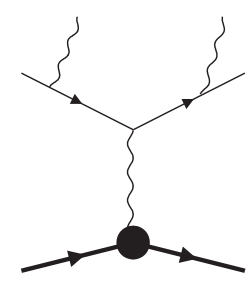

(b)

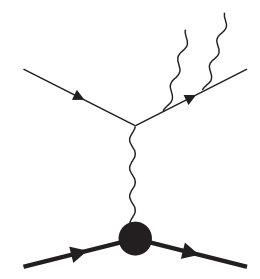

(c)

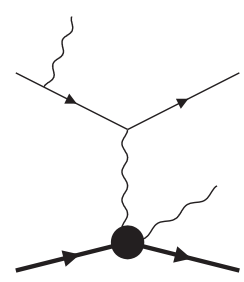

(d)

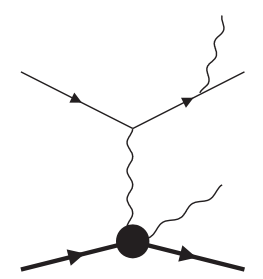

(e)
FIG. 2. Feynman graphs with two real photons in the final state: both photons produced by leptons (a), (b), and (c) and by leptons and hadrons (d) and (e).

$\mathcal{M}_{2 \gamma}^{2}=\sum_{i=1}^{6}\left[\mathcal{M}_{i}^{l l}\left(\mathcal{M}_{1}^{l h}+\mathcal{M}_{2}^{l h}\right)^{\dagger}+\left(\mathcal{M}_{1}^{l h}+\mathcal{M}_{2}^{l h}\right) \mathcal{M}_{i}^{l l \dagger}\right]$.

Six matrix elements of the process with the emission of the additional photon correspond to graphs in Figs. 2(a)-2(c): $\mathcal{M}_{1-6}^{l l}=e^{4} t^{-1} J_{\mu}^{h} J_{1-6, \mu}$. The quantities $J_{1-6, \mu}$ are defined by Eq. (22) of [9]:

$$
\begin{aligned}
& J_{1 \mu}=\bar{u}_{2} \gamma_{\mu} \frac{\hat{k}_{1}-\hat{\kappa}+m}{-2 \kappa k_{1}+V^{2}} \hat{\epsilon}_{2} \frac{\hat{k}_{1}-\hat{\kappa}_{1}+m}{-2 k_{1} \kappa_{1}} \hat{\epsilon}_{1} u_{1}, \\
& J_{2 \mu}=\bar{u}_{2} \gamma_{\mu} \frac{\hat{k}_{1}-\hat{\kappa}+m}{-2 \kappa k_{1}+V^{2}} \hat{\epsilon}_{1} \frac{\hat{k}_{1}-\hat{\kappa}_{2}+m}{-2 k_{1} \kappa_{2}} \hat{\epsilon}_{2} u_{1}, \\
& J_{3 \mu}=\bar{u}_{2} \hat{\epsilon}_{2} \frac{\hat{k}_{2}+\hat{\kappa}_{2}+m}{2 k_{2} \kappa_{2}} \hat{\epsilon}_{1} \frac{\hat{k}_{2}+\hat{\kappa}+m}{2 \kappa k_{2}+V^{2}} \gamma_{\mu} u_{1}, \\
& J_{4 \mu}=\bar{u}_{2} \hat{\epsilon}_{1} \frac{\hat{k}_{2}+\hat{\kappa}_{1}+m}{2 k_{2} \kappa_{1}} \hat{\epsilon}_{2} \frac{\hat{k}_{2}+\hat{\kappa}+m}{2 \kappa k_{2}+V^{2}} \gamma_{\mu} u_{1}, \\
& J_{5 \mu}=\bar{u}_{2} \hat{\epsilon}_{1} \frac{\hat{k}_{2}+\hat{\kappa}_{1}+m}{2 k_{2} \kappa_{1}} \gamma_{\mu} \frac{\hat{k}_{1}-\hat{\kappa}_{2}+m}{-2 k_{1} \kappa_{2}} \hat{\epsilon}_{2} u_{1}, \\
& J_{6 \mu}=\bar{u}_{2} \hat{\epsilon}_{2} \frac{\hat{k}_{2}+\hat{\kappa}_{2}+m}{2 k_{2} \kappa_{2}} \gamma_{\mu} \frac{\hat{k}_{1}-\hat{\kappa}_{1}+m}{-2 k_{1} \kappa_{1}} \hat{\epsilon}_{1} u_{1},
\end{aligned}
$$

where $V^{2}=\kappa^{2}=\left(\kappa_{1}+\kappa_{2}\right)^{2}$. The quantity $V^{2}$ has the meaning of missing mass squared in the experimental design when only charge particles (electron and proton) are detected and/or used for reconstruction of kinematical variables.

Matrix elements with emissions of one photon from the lepton line and one photon from the hadron line [Figs. 2(d) and $2(e)]$ are

$$
\begin{aligned}
\mathcal{M}_{1}^{l h}+\mathcal{M}_{2}^{l h}= & e^{4} \epsilon_{1}^{\alpha} \epsilon_{2}^{\beta}\left(\frac{J_{\mu \alpha}^{\mathrm{BH}}\left(k_{1}, k_{2}, \kappa_{1}\right) T_{\beta \mu}\left(\kappa_{2}\right)}{Q^{2}+2 q \kappa_{1}}\right. \\
& \left.+\frac{J_{\mu \beta}^{\mathrm{BH}}\left(k_{1}, k_{2}, \kappa_{2}\right) T_{\alpha \mu}\left(\kappa_{1}\right)}{Q^{2}+2 q \kappa_{2}}\right) .
\end{aligned}
$$

The matrix element squares (17) has four terms with denominators containing $\kappa_{1,2} k_{1}\left(s\right.$-peak) and $\kappa_{1,2} k_{2}(p$ peak):

$$
\mathcal{M}_{2 \gamma}^{2}=\mathcal{M}_{1 s}^{2}+\mathcal{M}_{1 p}^{2}+\mathcal{M}_{2 s}^{2}+\mathcal{M}_{2 p}^{2},
$$

where indices correspond to the unobserved photon, e.g., $1 s$ means that the photon with momentum $\kappa_{1}$ is unobserved and in the $s$-peak. Just these four terms contribute to the cross section in the leading approximation. Each of them (i.e., $1 / k_{1} \kappa_{1}, 1 / k_{1} \kappa_{2}, 1 / k_{2} \kappa_{1}$, or $\left.1 / k_{2} \kappa_{2}\right)$ contains the first order pole which can be extracted if to put vectors $\kappa_{1}$ and $\kappa_{2}$ in the peak and use $m \rightarrow 0$ in the coefficient at each respective pole. Practically the terms are calculated by using the following substitution: $\kappa_{1}=\left(1-z_{1}\right) k_{1}, \kappa_{1}=\left(1 / z_{2}-1\right) k_{2}$, $\kappa_{2}=\left(1-z_{1}\right) k_{1}$ and $\kappa_{2}=\left(1 / z_{2}-1\right) k_{2}$ for $\mathcal{M}_{1 s}^{2}, \mathcal{M}_{1 p}^{2}$, $\mathcal{M}_{2 s}^{2}$ and $\mathcal{M}_{2 p}^{2}$, respectively. The use of these formulas means putting the angular components of the vectors $\kappa_{1}$ and $\kappa_{2}$ to be equal of respective angular components of vectors $k_{1}$ and $k_{2}$ in numerators of all terms in the right-hand side of (20) $\mathcal{M}_{1,2 s, p}^{2}$, keeping the last component (i.e., energy of $\kappa_{1}$ and $\kappa_{2}$ ) unfixed. The variables $z_{1,2}$ represent the energyrelated components of the vectors and can be related to $V^{2}$ as

$$
z_{1}=1-\frac{V^{2}}{w}, \quad z_{2}=\frac{u}{u+V^{2}},
$$

where $w=2 k_{1}\left(p+q-p^{\prime}\right)$ and $u=2 k_{2}\left(p+q-p^{\prime}\right)$.

The calculation of $\mathcal{M}_{1 s}^{2}$ is similar to that considered in [9] but there are new technical issues because of the different structure of the matrix element squared. Only $\mathcal{M}_{1}^{l l}, \mathcal{M}_{6}^{l l}$, and $\mathcal{M}_{1}^{\text {lh }}$ can have the pole $1 / k_{1} \kappa_{1}$ through contributions from $J_{1 \mu}, J_{6 \mu}$ and $J_{\mu \alpha}^{\mathrm{BH} 1}$ that are reduced to

$$
\begin{aligned}
J_{1 \mu} & \approx \frac{k_{1} \epsilon_{1}}{2\left(k_{1} \kappa_{2}\right)\left(k_{1} \kappa_{1}\right)} \bar{u}_{2} \gamma_{\mu}\left(z_{1} \hat{k}_{1}-\hat{\kappa}_{2}\right) \hat{\epsilon}_{2} u_{1}, \\
J_{6 \mu} & \approx-\frac{z_{1} k_{1} \epsilon_{1}}{2\left(k_{2} \kappa_{2}\right)\left(k_{1} \kappa_{1}\right)} \bar{u}_{2} \hat{\epsilon}_{2}\left(\hat{k}_{2}+\hat{\kappa}_{2}\right) \gamma_{\mu} u_{1}, \\
J_{\mu \alpha}^{\mathrm{BH} 1}\left(k_{1}, \kappa_{1}\right) & \approx-\frac{z_{1} k_{1 \alpha}}{k_{1} \kappa_{1}} \bar{u}_{2} \gamma_{\mu} u_{1} .
\end{aligned}
$$

The convolution of the sum $\mathcal{M}_{1}^{l l}+\mathcal{M}_{6}^{l l}$ with $\mathcal{M}_{2}^{l h}$ contains the infrared divergence,

$$
\begin{gathered}
\left(\mathcal{M}_{1}^{l l}+\mathcal{M}_{6}^{l l}\right) \mathcal{M}_{2}^{l h \dagger}+\mathcal{M}_{2}^{l h}\left(\mathcal{M}_{1}^{l l}+\mathcal{M}_{6}^{l l}\right)^{\dagger} \\
=\frac{4 \pi \alpha}{\left(1-z_{1}\right) \kappa_{1} k_{1}} \mathcal{M}_{1 \gamma}^{2}\left(z_{1} k_{1}, k_{2}, \kappa_{2}\right),
\end{gathered}
$$

at $z_{1} \rightarrow 1$, while the convolution of this sum with $\mathcal{M}_{1}^{l h}$,

$$
\begin{gathered}
\left(\mathcal{M}_{1}^{l l}+\mathcal{M}_{6}^{l l}\right) \mathcal{M}_{1}^{l h \dagger}+\mathcal{M}_{1}^{l h}\left(\mathcal{M}_{1}^{l l}+\mathcal{M}_{6}^{l l}\right)^{\dagger} \\
=\frac{4 \pi \alpha\left(1-z_{1}\right)}{z_{1} \kappa_{1} k_{1}} \mathcal{M}_{1 \gamma}^{2}\left(z_{1} k_{1}, k_{2}, \kappa_{2}\right),
\end{gathered}
$$

does not. 
The convolution of the other terms $\mathcal{M}_{2-5}^{l l}$ with $\mathcal{M}_{1}^{\text {lh }}$ results in the term also containing the infrared divergence:

$$
\sum_{i=2}^{5}\left[\mathcal{M}_{i}^{l l} \mathcal{M}_{1}^{l h \dagger}+\mathcal{M}_{1}^{l h} \mathcal{M}_{i}^{l l \dagger}\right]=\frac{4 \pi \alpha \mathcal{M}_{1 \gamma}^{2}\left(z_{1} k_{1}, k_{2}, \kappa_{2}\right)}{\left(1-z_{1}\right) \kappa_{1} k_{1}} .
$$

All other convolutions do not have contributions to the cross sections in the leading log approximation.

The resulting expressions for the terms in (20) are

$$
\begin{aligned}
\mathcal{M}_{1 s}^{2} & =\frac{4 \pi \alpha}{\kappa_{1} k_{1}} \frac{\left(1+z_{1}^{2}\right)}{z_{1}\left(1-z_{1}\right)} \mathcal{M}_{1 \gamma}^{2}\left(z_{1} k_{1}, k_{2}, \kappa_{2}\right), \\
\mathcal{M}_{1 p}^{2} & =\frac{4 \pi \alpha}{\kappa_{1} k_{2}} \frac{\left(1+z_{2}^{2}\right)}{\left(1-z_{2}\right)} \mathcal{M}_{1 \gamma}^{2}\left(k_{1}, \frac{k_{2}}{z_{2}}, \kappa_{2}\right), \\
\mathcal{M}_{2 s}^{2} & =\frac{4 \pi \alpha}{\kappa_{2} k_{1}} \frac{\left(1+z_{1}^{2}\right)}{z_{1}\left(1-z_{1}\right)} \mathcal{M}_{1 \gamma}^{2}\left(z_{1} k_{1}, k_{2}, \kappa_{1}\right), \\
\mathcal{M}_{2 p}^{2} & =\frac{4 \pi \alpha}{\kappa_{2} k_{2}} \frac{\left(1+z_{2}^{2}\right)}{\left(1-z_{2}\right)} \mathcal{M}_{1 \gamma}^{2}\left(k_{1}, \frac{k_{2}}{z_{2}}, \kappa_{1}\right) .
\end{aligned}
$$

The integration over angular variables results in

$$
\begin{aligned}
& \int \frac{d \Gamma}{\kappa_{1} k_{1}}=d \Gamma_{0} \frac{L}{8 \pi^{2} w} d V^{2}, \\
& \int \frac{d \Gamma}{\kappa_{1} k_{2}}=d \Gamma_{0} \frac{L}{8 \pi^{2} u} d V^{2} .
\end{aligned}
$$

Thus, the matrix elements squared of the process with two real photons in the final state is expressed in terms of the BH/DVCS matrix element squared in the same way as for the $\mathrm{BH}$ process [9]. The phase space parametrization is also independent on the dynamics of the process and its parametrization obtained for the pure $\mathrm{BH}$ process is applicable for the contribution from the BH and DVCS interference as well [9]. Therefore, the cross section for radiative correction to the interference of $\mathrm{BH}$ and DVCS amplitudes keeps the same form:

$$
\begin{gathered}
\sigma_{s}\left(S, x, Q^{2}, t, \phi\right)=\frac{\alpha}{2 \pi} L \int_{z_{1}^{m}}^{1} d z_{1} \frac{1+z_{1}^{2}}{1-z_{1}} K_{s}\left(z_{1}\right) \sigma_{1 \gamma}\left(z_{1}\right), \\
\sigma_{p}\left(S, x, Q^{2}, t, \phi\right)=\frac{\alpha}{2 \pi} L \int_{z_{2}^{m}}^{1} d z_{2} \frac{1+z_{2}^{2}}{1-z_{2}} K_{p}\left(z_{2}\right) \sigma_{1 \gamma}\left(z_{2}\right) .
\end{gathered}
$$

Here $z_{1,2}$ in brackets means that the cross section needs to be taken in a shifted kinematics, i.e.,

$$
\begin{aligned}
& \sigma_{1 \gamma}\left(z_{1}\right)=\sigma_{1 \gamma}\left(z_{1} S, x_{s}, z_{1} Q^{2}, t, \bar{\phi}_{s}\right), \\
& \sigma_{1 \gamma}\left(z_{2}\right)=\sigma_{1 \gamma}\left(S, x_{p}, z_{2}^{-1} Q^{2}, t, \bar{\phi}_{p}\right)
\end{aligned}
$$

and

$$
K_{s}\left(z_{1}\right)=\frac{x_{s}^{2} \sin \theta_{s}^{\prime}}{x^{2} \mathcal{D}_{0 s}^{1 / 2}}, \quad K_{p}\left(z_{2}\right)=\frac{x_{p}^{2} \sin \theta_{p}^{\prime}}{z_{2} x^{2} \mathcal{D}_{0 p}^{1 / 2}},
$$

where $x_{s}=z_{1} Q^{2} /\left(z_{1} S-X\right)$ and $x_{p}=Q^{2} /\left(z_{2} S-X\right)$ are Bjorken $x$ in shifted kinematics; $\mathcal{D}_{0}, \sin \theta^{\prime}$ and $\bar{\phi}$ are given in [9] by Eqs. (38) and (40); the subscript ( $s$ or $p$ ) explicitly indicates the type of kinematics for which these quantities have to be calculated.

The lower limits of integration in (28) are defined by the substitutional in Eq. (21), the maximal missing mass squared

$$
V_{\max }^{2}=(2 M)^{-1}\left(\sqrt{\lambda_{Y}} \sqrt{\lambda_{t}}+S_{x} t\right)-Q^{2}+t
$$

with $\lambda_{t}=t\left(t-4 M^{2}\right)$ and reads as

$$
\begin{aligned}
& z_{1}^{m}=\frac{t\left(X-2 M^{2}\right)+\sqrt{\lambda_{t} / \lambda_{Y}}\left(X S_{x}-2 M^{2} Q^{2}\right)}{S t-2 M^{2} Q^{2}+\sqrt{\lambda_{t} / \lambda_{Y}}\left(S S_{x}+2 M^{2} Q^{2}\right)}, \\
& z_{2}^{m}=\frac{X t-2 M^{2} Q^{2}+\sqrt{\lambda_{t} / \lambda_{Y}}\left(X S_{x}-2 M^{2} Q^{2}\right)}{t\left(S+2 M^{2}\right)+\sqrt{\lambda_{t} / \lambda_{Y}}\left(S S_{x}+2 M^{2} Q^{2}\right)} .
\end{aligned}
$$

The relationship between $z_{1,2}$ and $V^{2}$ is illustrated in Fig. 3. In most cases the relation between them is unambiguous as shown in Fig. 3(a). However there are situations (for $\cos \phi<0$ ) when the curves $z_{1,2}\left(V^{2}\right)$ have a minimum [Fig. 3(b)]. In this case an additional contribution, $\sigma_{\text {add }}$, reflecting the area between two points of intersections between the curve $z_{1,2}\left(V^{2}\right)$ and the line $z_{1,2}=z_{1,2}^{m}$ can to be presented as a separate contribution to the cross section. The explicit form of $\sigma_{\text {add }}$ is given in the Appendix.

The integrals in (28) are divergent at the upper integration limit because of the infrared divergence, which is
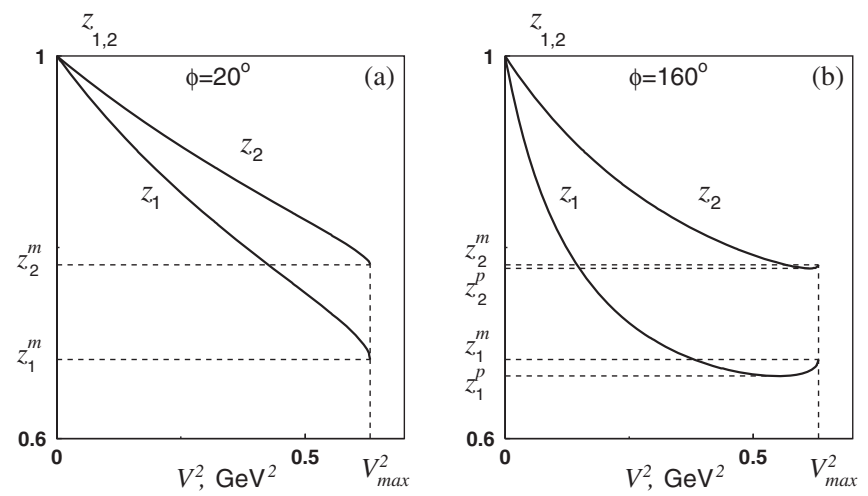

FIG. 3. The dependence of $z_{1}$ and $z_{2}$ on $V^{2}$ for $\cos (\phi)>0$ (a) and $\cos (\phi)<0$ (b). Other kinematical variables used for this example were $x=0.175, Q^{2}=1 \mathrm{GeV}^{2}, t=-0.1 \mathrm{GeV}^{2}$, and $E_{\text {beam }}=5.75 \mathrm{GeV}$. In plot (b), the curves $z_{1,2}\left(V^{2}\right)$ cross the lines $z_{1,2}=z_{1,2}^{m}$ at $V^{2}=V_{1 s, p}^{2}$ and reach their minimum values at $V^{2}=$ $V_{2 s, p}^{2}$ such that $V_{1 s, p}^{2}<V_{2 s, p}^{2}<V_{\max }^{2}$. The explicit expressions for $z_{1,2}^{p}$ and $V_{1,2 s, p}^{2}$ are given in the Appendix. 


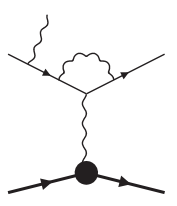

(a)

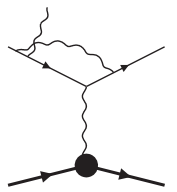

(e)

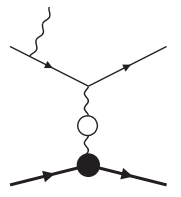

(i)

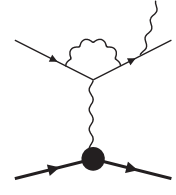

(b)

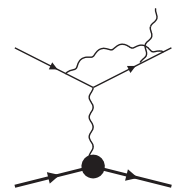

(f)

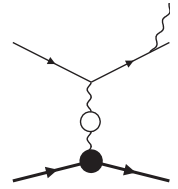

(j)

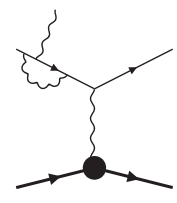

(c)

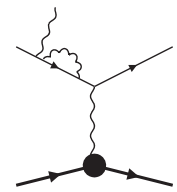

(g)

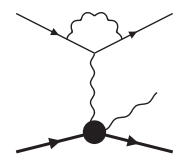

(k)

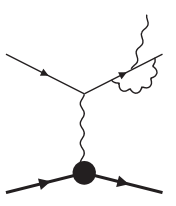

(d)

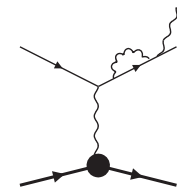

(h)

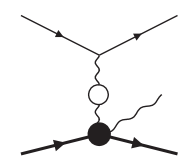

(1)
FIG. 4. The one-loop Feynman graphs for the BH (a-h) and DVCS (k) amplitudes containing the infrared divergence and the graphs for the vacuum polarization (i), (j), and (l).

canceled by adding the contribution of loops and soft photon emission [8] represented by the Feynman graphs in Figs. 4(a) $-4(\mathrm{~h})$ and $4(\mathrm{k})$. The result for the infrared free contribution is

$$
\frac{\alpha}{\pi}\left(\delta_{\text {inf }}+\delta_{\text {fin }}\right) \sigma_{1 \gamma}+\sigma_{s}^{F}+\sigma_{p}^{F}+\sigma_{\text {add }},
$$

where

$$
\begin{aligned}
& \delta_{\text {fin }}=\frac{L}{4}\left(z_{1}^{m}\left(2+z_{1}^{m}\right)+z_{2}^{m}\left(2+z_{2}^{m}\right)\right), \\
& \delta_{\text {inf }}=L\left(\log \left(1-z_{1}^{m}\right)+\log \left(1-z_{2}^{m}\right)\right)
\end{aligned}
$$

and

$$
\begin{gathered}
\sigma_{s}^{F}=\frac{\alpha L}{2 \pi} \int_{z_{1}^{m}}^{1} d z_{1} \frac{1+z_{1}^{2}}{1-z_{1}}\left(K_{s}\left(z_{1}\right) \sigma_{1 \gamma}\left(z_{1}\right)-\sigma_{1 \gamma}\right), \\
\sigma_{p}^{F}=\frac{\alpha L}{2 \pi} \int_{z_{2}^{m}}^{1} d z_{2} \frac{1+z_{2}^{2}}{1-z_{2}}\left(K_{p}\left(z_{2}\right) \sigma_{1 \gamma}\left(z_{2}\right)-\sigma_{1 \gamma}\right) .
\end{gathered}
$$

The experimental cuts on missing mass squared $V_{\text {cut }}^{2}$ or maximal photon energy can be incorporated by the following replacements:

$$
K_{s, p}\left(z_{1,2}\right) \rightarrow \theta\left(z_{1,2}-z_{1,2}^{c}\right) K_{s, p}\left(z_{1,2}\right) .
$$

Here $z_{1,2}^{c}$ reflect the restrictions on the energy of the hard photon or missing mass squared. The relation between these variables are given in Eq. (A6) of the Appendix.

The total lowest order RC is

$\sigma_{\mathrm{RC}}=\frac{\alpha}{\pi}\left(\delta_{\mathrm{vac}}+\delta_{\mathrm{inf}}+\delta_{\mathrm{fin}}\right) \sigma_{1 \gamma}+\sigma_{s}^{F}+\sigma_{p}^{F}+\sigma_{\mathrm{add}}$.
Here $\delta_{\text {vac }}$ reflects the contribution of vacuum polarization, i.e., the Feynman graphs in Figs. 4(i), 4(j), and 4(1). Specifically, $\Pi(t)=\alpha /(2 \pi) \delta_{\mathrm{vac}}$ and $\delta_{\mathrm{vac}}$ is the contribution of vacuum polarization by leptons and hadrons calculated as in [12] [see Eq. (21) and discussion before Eq. (20)]. Formally, the expression for the observed cross section coincides with the cross section for the $\mathrm{BH}$ process obtained in [9] [expression (48)]. The higher order corrections can be included in the style of (51) or (52) of Ref. [9].

The behavior of the cross section for $t$ close to kinematical bounds (i.e., in the region where $t \sim t_{1}$ and $t \sim t_{2}$ ) deserves special attention. The quantity $\delta_{\text {inf }}$ in (34) becomes infinite when $t \rightarrow t_{1}$ or $t \rightarrow t_{2}$. In this limit $z_{1}^{m}=1$ and $z_{2}^{m}=1$. The source of the occurrence of the divergence is known [13]. The divergence is canceled by taking into account multiple soft photon emission. We follow the so-called exponentiation procedure suggested in [14]:

$$
\begin{aligned}
(1 & \left.+\frac{\alpha}{\pi}\left(\delta_{\mathrm{vac}}+\delta_{\mathrm{inf}}+\delta_{\mathrm{fin}}\right)\right) \\
& \rightarrow \exp \left(\frac{\alpha}{\pi} \delta_{\text {inf }}\right)\left(1+\frac{\alpha}{\pi}\left(\delta_{\mathrm{vac}}+\delta_{\mathrm{fin}}\right)\right),
\end{aligned}
$$

such that the observed cross section becomes

$$
\begin{aligned}
\sigma_{\mathrm{obs}}= & \exp \left(\frac{\alpha}{\pi} \delta_{\text {inf }}\right)\left(1+\frac{\alpha}{\pi}\left(\delta_{\mathrm{vac}}+\delta_{\text {fin }}\right)\right) \sigma_{1 \gamma} \\
& +\sigma_{s}^{F}+\sigma_{p}^{F}+\sigma_{\text {add }} .
\end{aligned}
$$

After this procedure the observed cross section vanishes at the kinematical bounds on $t$.

This result allows us to construct a Monte Carlo generator of the events with one or two photons in the final state. Such a generator is reasonable to construct for the total cross section that contains three contributions coming from a pure $\mathrm{BH}$ process (BH amplitude squared), pure DVCS process (DVCS amplitude squared), and the interference between BH and DVCS amplitudes. RC given by Eqs. (28)-(35) and (A1) is applicable for the interference term and pure $\mathrm{BH}$ process [9]. The derived formulas are also applicable for pure DVCS contribution, so, alternatively, the pure DVCS term can be optionally included in event generation. Although the result for the pure DVCS contribution is not proved, we can provide some arguments why the formulas are valid for this contribution as well. The amplitudes contributed to the RC to the pure DVCS cross section include one photon radiated by leptons and one photon radiated by hadrons: the standard leading $\log$ in the case of one photon was calculated many times resulting in the same $z_{1,2}$-dependent coefficient exactly as in (28) and the remaining coefficients came from twophoton phase space parametrization.

To have an opportunity to simulate the specific contributions we must represent the observed cross section as a sum of positively definite contributions. Because of the last terms in (35), i.e., the terms containing $\sigma_{1 \gamma}$, the contributions $\sigma_{s, p}^{F}$ are not positively definite. These terms can be decomposed using 


$$
\begin{aligned}
& \int_{z_{1}^{m}}^{1} d z_{1} \frac{1+z_{1}^{2}}{1-z_{1}}\left(K_{s}\left(z_{1}\right) \sigma_{1 \gamma}\left(z_{1}\right)-\sigma_{1 \gamma}\right) \\
& =\int_{z_{1}^{m}}^{1-\frac{\Delta}{E}} d z_{1} \frac{1+z_{1}^{2}}{1-z_{1}} K_{s}\left(z_{1}\right) \sigma_{1 \gamma}\left(z_{1}\right)-\sigma_{1 \gamma} \int_{z_{1}^{m}}^{1-\frac{\Delta}{E}} d z_{1} \frac{1+z_{1}^{2}}{1-z_{1}} \\
& \quad+\int_{1-\frac{\Delta}{E}}^{1} d z_{1} \frac{1+z_{1}^{2}}{1-z_{1}}\left(K_{s}\left(z_{1}\right) \sigma_{1 \gamma}\left(z_{1}\right)-\sigma_{1 \gamma}\right)
\end{aligned}
$$

and similarly for $\sigma_{p}^{F}$. The quantity $\Delta$ is defined as a minimal energy of the photon we want to generate (i.e., calorimeter

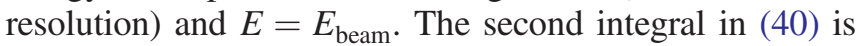
calculated analytically. The third integral vanishes for $\Delta \rightarrow 0$; therefore, it could be neglected (or kept and added to the contribution of the one-gamma contribution). The calculation results in

$$
\begin{aligned}
& \sigma_{s}^{F}=\sigma_{s}(\Delta)+\delta_{s}(\Delta) \sigma_{1 \gamma}, \\
& \sigma_{p}^{F}=\sigma_{p}(\Delta)+\delta_{p}(\Delta) \sigma_{1 \gamma},
\end{aligned}
$$

where $\sigma_{s, p}(\Delta)$ represent the first term in (40).

Combining all together, we have for the cross section with the lowest order RC

$\sigma_{\mathrm{obs}}=\left(1+\frac{\alpha}{\pi}\left(\delta_{\mathrm{vac}}+\delta(\Delta)\right)\right) \sigma_{1 \gamma}+\sigma_{s}(\Delta)+\sigma_{p}(\Delta)+\sigma_{\mathrm{add}}$

where

$$
\begin{aligned}
\delta(\Delta) & =\delta_{s}(\Delta)+\delta_{p}(\Delta)+\delta_{\text {inf }}+\delta_{\text {fin }} \\
& =L\left(\frac{3}{2}+\log \left(\frac{4 M^{2} \Delta^{2}}{S X}\right)\right) .
\end{aligned}
$$

Each contribution in (42) is positively definite. The price for this representation is the dependence on $\Delta$.

The event is generated for a kinematical point $x, Q^{2}, t$, and $\phi$ according to (42), and the electron azimuthal angle $\left(\phi_{e}\right)$ is simulated uniformly. Then the probabilities of all three channels: nonradiated (i.e., no an additional radiated photon), radiated in $s$-peak, and radiated in $p$-peak are calculated as

$$
\begin{aligned}
& p_{\text {nonrad }}=\left(1+\frac{\alpha}{\pi}\left(\delta_{\mathrm{vac}}+\delta(\Delta)\right)\right) \frac{\sigma_{1 \gamma}}{\sigma_{\mathrm{obs}}}, \\
& p_{s \text {-peak }}=\frac{\sigma_{s}(\Delta)}{\sigma_{\mathrm{obs}}} \\
& p_{p \text {-peak }}=\frac{\sigma_{p}(\Delta)}{\sigma_{\mathrm{obs}}} .
\end{aligned}
$$

The scattering channel is generated according to these three probabilities. If the event with one photon in the final state is chosen then no additional variables are needed to be simulated. If the two-photon event in $s$-peak or $p$-peak is chosen, then the three kinematical variables of an additional photon are needed to be simulated. The photon energy is simulated through the variable $z_{1}$ or $z_{2}$ (for $s$ - and $p$-peaks respectively) according to their distributions in the integrand of $\sigma_{s}(\Delta)$ and $\sigma_{p}(\Delta)$. The photon angles are simulated in $s$ - or $p$-peaks; i.e., the photon angles become equal to the angles of the initial or final lepton. Note, in (44) the components of $\sigma_{\text {add }}$ that correspond to $s$ - and $p$-peaks are included in the definition of $\sigma_{s}(\Delta)$ and $\sigma_{p}(\Delta)$.

\section{CODES FOR NUMERICAL CALCULATION OF RC IN A KINEMATICAL POINT AND MONTE CARLO GENERATOR}

The results presented in previous section allows us to create a code for numerical calculation of $\mathrm{RC}$ in a kinematical point (i.e., for specific $x, Q^{2}, t, \phi$, and beam energy) and the respective Monte Carlo generator [15].

The Fortran code is called DVCSLL. Special keys allow us to choose the part of the cross section (i.e., BH only, BH-DVCS interference, etc.), the approximation for hadronic part (exact for $\mathrm{BH}$ only or $\mathrm{BMK}$ ), electron and proton polarizations, accuracies of integration, and the values of kinematical variables and the cut on missing mass. The Monte Carlo generator GenDVCSLL works as a slave system; i.e., it generates one event for a kinematical point externally given. An additional parameter for the Monte Carlo generator is $\Delta$.

Thus, DVCSLL is the code to calculate $\mathrm{RC}$ to the cross section of photon electroproduction (i.e., pure $\mathrm{BH}$ process, its interference with DVCS amplitude, and optionally, pure DVCS contribution) in leading approximation. The specific features of the approach and properties of the resulting expressions include (i) the cross section of the lowest order in a shifted kinematical point is factorized in the integrand, (ii) no additional assumptions about hadronic structure (except those necessary to calculate the cross section with one photon in the final state) are required, (iii) cases of longitudinal and transverse target polarization are included, (iv) higher order corrections are included using a procedure of exponentiation (alternative approach in terms of electron structure functions was used in [8,9]), (v) a cut on missing energy is implemented, and (vi) numeric integration over the photon phase space represented by $\left(x, Q^{2}, t, \phi\right)$ is implemented. BMK approximation is used to describe the hadronic structure for DVCS. Note that only the leading log correction is implemented. The next-to-leading $\mathrm{RC}$ for the $\mathrm{BH}$ cross section of polarized particles is calculated in [10].

The leading log accuracy is the main uncertainty of theoretical calculation. Other theoretical uncertainties the 
researcher has to keep in mind include (i) higher order corrections through exponentiation procedure (not so high effect is expected), (ii) accuracy of numeric integration (largely under control), and (iii) approximations made when experimental cuts are implemented (could be tested) and finally resolved (using Monte Carlo generators). Besides, there are physical contributions not taken into account yet, e.g., the pentagon (or five-point) diagrams, i.e., the box diagram with a photon emission from the lepton line. Another type of uncertainty is the model dependence, such as the model for the nucleon form factors (essential effect is not expected, but needed to be checked for each specific data analysis) and the BMK approximation for RC to DVCS.

The design of the Monte Carlo generator BHRadgen is as follows. The input required by the generator is (i) the four kinematical variables $x, Q^{2}, t$, and $\phi$; (ii) the value of $\Delta$; (iii) beam energy; and (iv) the value of $V_{\text {cut }}^{2}$. The output is (i) generated channel of scattering for an event, i.e., radiated (two photons in final state) or nonradiated (one photon in final state), (ii) three additional kinematical variables (to describe an additional photon) generated for the radiated event, and (iii) the cross section of RC for any event. The cross sections and distributions over additional kinematical variables are calculated for the given kinematical point $\left(x, Q^{2}, t\right.$, and $\left.\phi\right)$. Then any number of events are simulated using this information. If simulation of many events is required for a certain kinematical point, then the program is efficient. However, the computation is not so fast if the point needs to be simulated for each event. Approaches to accelerate generation of an event could include (i) a look-up table storing information about additional photon energies and angles in a kinematical region, (ii) relaxation of requirements to the accuracy of Monte Carlo integration, and (iii) using a numeric approach for integration and calculation of distribution over additional photonic variables. Collinear kinematics is used for simulation of photonic angles. Instead, the distribution can be used from the integrand over photonic angles. The calculation is based on the leading log approximation. Next-to-leading corrections can be implemented using results for the RC calculation with the next-to-leading accuracy [10]. In this case new analytical results for the distribution over additional photonic variables need to be obtained and implemented. The current code was obtained using the results integrated over two angles of an additional photon. Exact formulas are implemented for the BH only. Contributions of DVCS are calculated in the BMK approximation.

\section{NUMERIC ANALYSIS}

The experimental access to characteristics of the DVCS amplitudes is provided by the measurement of the beamspin asymmetry (11). The observed asymmetry can be represented as

$$
A=A_{1 \gamma} \frac{\delta_{p}}{\delta_{u}},
$$

where $\delta_{u, p}$ are RC factors for unpolarized (i.e., presented in the denominator of $A_{1 \gamma}$ ) and polarized (i.e., presented in the numerator of $A_{1 \gamma}$ ) parts of the cross section. The relative correction to asymmetry is defined as

$$
\delta_{A}=\frac{A-A_{1 \gamma}}{A_{1 \gamma}} .
$$

The results for $\phi$ - and $t$-dependencies are presented in Figs. 5 and 6, respectively. For Compton form factors, the model suggested by Korotkov and Nowak [16] is used. RC is much higher in the region of $90^{\circ}<\phi<270^{\circ}$ and small value of $-t$. It is clear that the largest contribution to RC comes from collinear kinematics when $w_{0}$ or $u_{0}$ is minimal. Minimal values of these quantities can be achieved in this region when $-t$ trends to its minimum values, $\cos \phi$ is negative, and the absolute minimum is of order $\mathrm{m}^{2}$ for $\phi=180^{\circ}$.
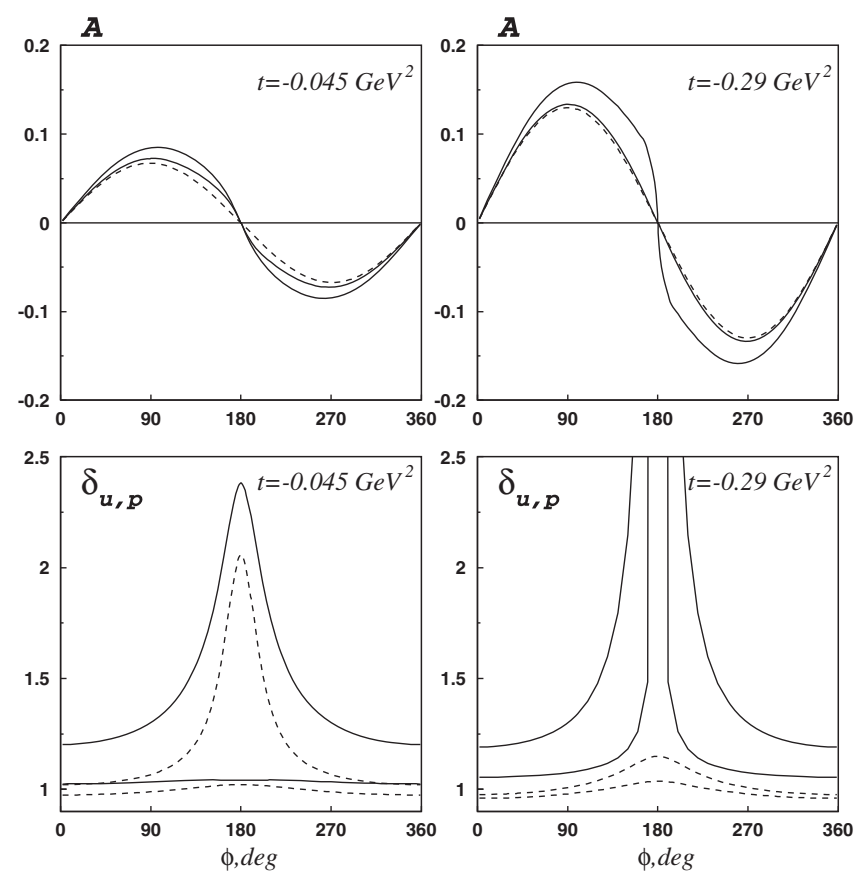

FIG. 5. The $\phi$-dependence of the asymmetry (upper) and $\mathrm{RC}$ factors (lower plots). The dashed curve at the upper plots gives the $\sigma_{1 \gamma}$ and the solid curve shows the observed cross sections with $V_{\text {cut }}^{2}=0.3 \mathrm{GeV}^{2}$ (the curve closer to dashed curve) and without cuts. Dashed and solid curves at the bottom plots show $\delta_{u, p}$ with and without the cut, respectively. The curves with higher values corresponds to $\delta_{p}$, i.e., $\delta_{p}>\delta_{u}$. Kinematical variables used for this example were $x=0.1$, $Q^{2}=2 \mathrm{GeV}^{2}$, and $E_{\text {beam }}=11 \mathrm{GeV}$. 

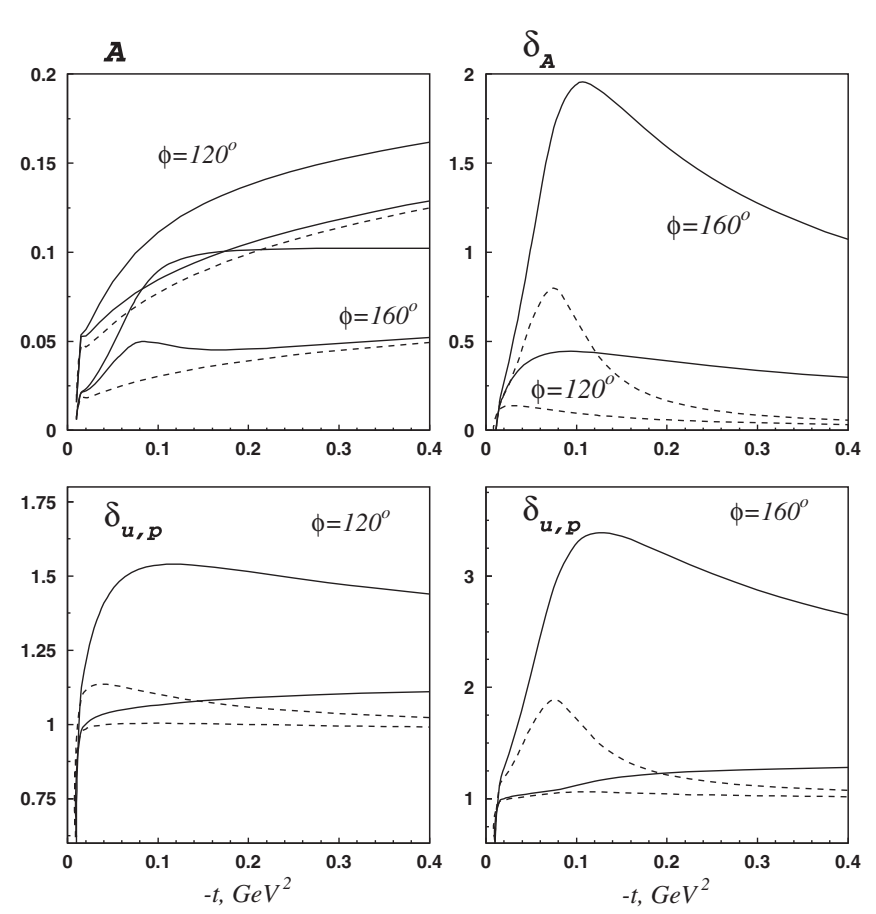

FIG. 6. The $t$-dependence of the asymmetry (upper left), RC to asymmetry (upper right), and RC factors (lower plots). Dashed curve for $A$ gives the $\sigma_{1 \gamma}$ and solid curved show the observed cross sections with $V_{\text {cut }}^{2}=0.3 \mathrm{GeV}^{2}$ (the curve closer to dashed curve) and without cuts. Dashed and solid curves at the other three plots show $\delta_{A, u, p}$ with and without the cut, respectively. Kinematical variables used for this example were $x=0.1$, $Q^{2}=2 \mathrm{GeV}^{2}$, and $E_{\text {beam }}=11 \mathrm{GeV}$.

\section{SUMMARY AND CONCLUSION}

In this paper we used the leading log approximation to calculate the radiative corrections to the cross sections of photon electroproduction induced by the interference between the Bethe-Heitler and deep virtual Compton scattering amplitudes. The calculated RC is valid for the experimental design when both final charged particles are detected and their momenta are used for reconstruction of kinematical variables in a bin $[3,4,6]$. The steps required for the calculation of $\mathrm{RC}$ to the pure $\mathrm{BH}$ process and interference of BH and DVCS amplitude are (i) matrix element squared, (ii) integration over loops and taking care on ultraviolet divergence (i.e., making the electron charge and mass renormalization), (iii) phase space parametrization and integration over a part of kinematical variables of an additional photon: the cross section is defined by four kinematical variables $\left(x, Q^{2}, t\right.$ and $\left.\phi\right)$ and the cross section with a two photon emitted is defined by seven kinematical variables, including the same four variables $\left(x, Q^{2}, t\right.$, and $\left.\phi\right)$ and three additional variables (the two-photon invariant mass squared $V^{2}$ and two angles of the photon pair), (iv) extraction and cancellation of the infrared divergence without making new assumptions, (v) implementation of a contribution of higher order corrections (calculated approximately), and (vi) coding the results to have, first, a program for RC calculation in a kinematical point $\left(x, Q^{2}, t\right.$, and $\left.\phi\right)$ and the Monte Carlo generator with the inclusion of RC contributions. The calculation in the leading log approximation resulted in (28). Adding the contribution of loops and canceling the infrared divergence results in (37). Finally, the exponentiation of multiple soft photons results in the final formula in (38). The calculation of the next-to-leading-order correction to the $\mathrm{BH}$ cross section is much more complicated [10]. The code created to calculate RC in a kinematical point based on these calculations is named DVCSLL. This code allows us to calculate RC to BH and DVCS process in leading approximation. Cases of longitudinal and transverse target polarization are included. Higher order corrections are included through exponentiation and potentially higher order corrections in terms of electron structure functions available for $\mathrm{BH}$ can be included for the interference of BH and DVCS as well. An opportunity to incorporate a cut on missing energy is implemented. The numeric integration over the phase space of an additional photon is implemented, and the BMK approximation [11] is used to describe hadronic structure for DVCS. The approach implemented in the Monte Carlo generator allowed for the generation of DVCS events and Monte Carlo integration over an experimental bin. For each event the Monte Carlo generator selects between radiated (two photons in final state) or nonradiated (one photon in final state) and if the radiated event is selected, three additional kinematical variables to describe an additional photon are generated. A numerical analysis of the $\mathrm{RC}$ to cross sections and asymmetries allowed us to conclude that the RC is under control and remaining uncertainties are due to model dependence and to the effects not taken into account yet (e.g., the pentagon diagrams).

\section{ACKNOWLEDGMENTS}

The authors are grateful to Harut Avakian and Volker Burkert for interesting discussions and comments. This work was supported by DOE Contract No. DE- AC0506OR23177, under which Jefferson Science Associates, LLC operates Jefferson Lab. The codes DVCSLL and GenDVCSLL are available by request [15].

\section{APPENDIX: EXPLICIT EXPRESSION FOR $\sigma_{\text {add }}$}

The analytical expression for the term $\sigma_{\text {add }}$ is

$$
\begin{aligned}
\sigma_{\text {add }}= & \frac{\alpha L}{2 \pi}\left[\int_{z_{1}^{p}}^{z_{1}^{m}} d z_{1} \frac{1+z_{1}^{2}}{1-z_{1}} \frac{\hat{\delta}_{s} \sin \theta_{s}^{\prime}+\tilde{\delta}_{s} \sin \tilde{\theta}_{s}^{\prime}}{\mathcal{D}_{0 s}^{1 / 2}}\left(\frac{x_{s}}{x}\right)^{2} \sigma_{1 \gamma}\left(z_{1}\right)\right. \\
& \left.+\int_{z_{2}^{p}}^{z_{2}^{m}} d z_{2} \frac{1+z_{2}^{2}}{z_{2}\left(1-z_{2}\right)} \frac{\hat{\delta}_{p} \sin \theta_{p}^{\prime}+\tilde{\delta}_{p} \sin \tilde{\theta}_{p}^{\prime}}{\mathcal{D}_{0 p}^{1 / 2}}\left(\frac{x_{p}}{x}\right)^{2} \sigma_{1 \gamma}\left(z_{2}\right)\right]
\end{aligned}
$$


for $\cos \phi<0$, and $\sigma_{\text {add }}=0$ for $\cos \phi \geq 0$. In (A1), $\sigma_{1 \gamma}\left(z_{1,2}\right)$ are defined by (29), $\sin \theta^{\prime}$ is given in [9] by Eq. (38) as one of solution of Eq. (35), and $\sin \tilde{\theta}_{s, p}^{\prime}$ represent other solution

$$
\sin \tilde{\theta}^{\prime}=-\frac{\cos \theta_{z} \sqrt{\mathcal{D}_{0}}+A \sin \theta_{z} \cos \phi}{\cos \theta_{z}^{2}+\sin ^{2} \theta_{z} \cos ^{2} \phi} .
$$

The lowest limits of integration in (A1) are defined as

$$
\begin{aligned}
z_{1,2}^{p}= & 1-4 \lambda_{Y} V_{+}^{2} V_{-}^{2}\left[\left(V_{-}^{2}-V_{+}^{2}\right) \sqrt{D_{s, p}}\right. \\
& \left.+\left(V_{+}^{2}+V_{-}^{2}\right) A_{2 s, p}+2 V_{+}^{2} V_{-}^{2}\left(\lambda_{Y}+S_{x} S_{p}\right)\right]^{-1},
\end{aligned}
$$

where

$$
\begin{aligned}
V_{ \pm}^{2} & =\frac{t S_{x} \pm \sqrt{\lambda_{t}} \sqrt{\lambda_{Y}}}{2 M^{2}}-Q^{2}+t, \\
D_{s, p} & =A_{1}^{2} V_{+}^{2} V_{-}^{2}+A_{2 s, p}^{2}, \\
A_{1} & =4 M \cos (\phi) \sqrt{Q^{2}\left(S X-M^{2} Q^{2}\right)-m^{2} \lambda_{Y}}, \\
A_{2 s} & =Q^{2}\left(S_{p}\left(S_{x}+2 t\right)-\lambda_{Y}\right)-t\left(\lambda_{Y}+S_{p} S_{x}\right), \\
A_{2 p} & =Q^{2}\left(S_{p}\left(S_{x}+2 t\right)+\lambda_{Y}\right)+t\left(\lambda_{Y}-S_{p} S_{x}\right) .
\end{aligned}
$$

The quantities $\hat{\delta}_{s, p}$ and $\tilde{\delta}_{s, p}$ are introduced to reflect experimental cuts on $V^{2}$ and therefore $z_{1,2}$. There are four cases for the cutting value of $V^{2}$, i.e., no cut and when $V_{\text {cut }}^{2}$ is between $V_{1 s, p}^{2}, V_{2 s, p}^{2}$ or $V_{\max }^{2}$ [Fig. 3(b)]. First, when no cut on missing mass square is used, $\hat{\delta}_{s, p}=\tilde{\delta}_{s, p}=1$. Second, if $V_{\text {cut }}^{2} \leq V_{1 s, p}^{2}$, then $\hat{\delta}_{s, p}=\tilde{\delta}_{s, p}=0$. Third, if $V_{1 s, p}^{2}<V_{\text {cut }}^{2}<$ $V_{2 s, p}^{2}$, then $\hat{\delta}_{s, p}=\theta\left(z_{1,2}-z_{1,2}^{c}\right)$ and $\tilde{\delta}_{s, p}=0$. Fourth, $V_{2 s, p}^{2}<V_{\text {cut }}^{2}<V_{\max }^{2}$ then $\hat{\delta}_{s, p}=1$ and $\tilde{\delta}_{s, p}=\theta\left(z_{1,2}^{c}-z_{1,2}\right)$. Formally, $\hat{\delta}_{s, p}$ and $\tilde{\delta}_{s, p}$ can be presented using a combined formula aggregating all four cases:

$$
\begin{aligned}
\hat{\delta}_{s, p}= & \theta\left(V_{\mathrm{cut}}^{2}-V_{1 s, p}^{2}\right) \theta\left(V_{2 s, p}^{2}-V_{\mathrm{cut}}^{2}\right) \theta\left(z_{1,2}-z_{1,2}^{c}\right) \\
& +\theta\left(V_{\mathrm{cut}}^{2}-V_{2 s, p}^{2}\right), \\
\tilde{\delta}_{s, p}= & \theta\left(V_{\mathrm{cut}}^{2}-V_{2 s, p}^{2}\right) \theta\left(z_{1,2}^{c}-z_{1,2}\right) .
\end{aligned}
$$

The restrictions on $z_{1,2}$ read

$$
\begin{aligned}
z_{1,2}^{c}= & 1-2 \lambda_{Y} V_{\mathrm{cut}}^{2}\left[A_{1} \sqrt{\left(V_{+}^{2}-V_{\mathrm{cut}}^{2}\right)\left(V_{\mathrm{cut}}^{2}-V_{-}^{2}\right)}\right. \\
& \left.+A_{2 s, p}+V_{\mathrm{cut}}^{2}\left(S_{p} S_{x}+\lambda_{q}\right)\right]^{-1}
\end{aligned}
$$

and the other quantities used in previous expressions are

$$
\begin{aligned}
& V_{1 s, p}^{2}=\frac{V_{+}^{2} D_{s, p}}{A_{1}^{2} V_{+}^{2}+A_{2 s, p}^{2}}<V_{\max }^{2}, \\
& V_{2 s, p}^{2}=\frac{2 V_{+}^{2} V_{-}^{2} \sqrt{D_{s, p}}}{\left(V_{+}^{2}+V_{-}^{2}\right) \sqrt{D_{s, p}}-A_{2 s, p}\left(V_{+}^{2}-V_{-}^{2}\right)} .
\end{aligned}
$$

[1] A. V. Belitsky and D. Müller, Phys. Rev. D 79, 014017 (2009).

[2] A. V. Belitsky and D. Müller, Phys. Rev. D 82, 074010 (2010).

[3] F. Girod et al. (CLAS Collaboration), Phys. Rev. Lett. 100, 162002 (2008).

[4] H. S. Jo et al. (CLAS Collaboration), Phys. Rev. Lett. 115, 212003 (2015).

[5] M. Defurne et al., Nat. Commun. 8, 1408 (2017).

[6] R. Akhunzyanov et al. (COMPASS Collaboration), arXiv: 1802.02739.

[7] M. Vanderhaeghen, J. M. Friedrich, D. Lhuillier, D. Marchand, L. Van Hoorebeke, and J. Van de Wiele, Phys. Rev. C 62, 025501 (2000).

[8] V. V. Bytev, E. A. Kuraev, and E. Tomasi-Gustafsson, Phys. Rev. C 77, 055205 (2008).
[9] I. Akushevich and A. Ilyichev, Phys. Rev. D 85, 053008 (2012).

[10] I. Akushevich, A. Ilyichev, and N. M. Shumeiko, Phys. Rev. D 90, 033001 (2014).

[11] A. V. Belitsky, D. Müller, and A. Kirchner, Nucl. Phys. B629, 323 (2002).

[12] I. Akushevich and N. Shumeiko, J. Phys. G 20, 513 (1994).

[13] D. Yennie, S. Frautschi, and H. Suura, Ann. Phys. 13, 379 (1961).

[14] N. M. Shumeiko, Sov. J. Nucl. Phys. 29, 807 (1979).

[15] The codes are available by request (email:ily@hep.by).

[16] V. A. Korotkov and W. D. Nowak, Eur. Phys. J. C 23, 455 (2002). 